\title{
Non-model segmentation of brain glioma tissues with the combination of DWI and fMRI signals
}

\author{
Min Lu', Xiaojie Zhang ${ }^{\mathrm{a}}$, Mingyu Zhang ${ }^{\mathrm{b}, \mathrm{c}}$, Hongyan Chen ${ }^{\mathrm{b}}$,Weibei Dou ${ }^{\mathrm{a},{ }^{*}}$, Shaowu Li ${ }^{\mathrm{c}}$ and \\ Jianping Dai ${ }^{\text {b,c }}$ \\ ${ }^{a}$ Department of Electronic Engineering, Tsinghua University, Beijing, 100084, China \\ ${ }^{b}$ Radiology Department of Beijing Tiantan Hospital, Capital Medical University, Beijing, 100050, \\ China \\ ${ }^{c}$ Beijing Neurosurgical Institute, Beijing, 100050, China
}

\begin{abstract}
For quantitative analysis of glioma, multimodal Magnetic Resonance Imaging (MRI) signals are required in combination to perform a complementary analysis of morphological, metabolic, and functional changes. Most of the morphological analyses are based on T1-weighted and T2-weighted signals, called traditional MRI. But more detailed information about tumorous tissues could not be explained. An information combination scheme of Diffusion-Weighted Imaging (DWI) and Blood-Oxygen-Level Dependent (BOLD) contrast Imaging is proposed in this paper. This is a non-model segmentation scheme of brain glioma tissues in a particular perspective of combining multi-parameters of DWI and BOLD contrast functional Magnetic Resonance Imaging (fMRI). Compared with traditional MRI, a promising advantage of our work is to provide an effective and adequate subdivision of the related pathological regions with glioma, by incorporating both knowledge of image graylevel and spatial structure. Furthermore, it is an automatic segmentation method without needs of parameter selection and model fitting for the extracted tissues. By the experiments in patients with glioma, the proposed method has achieved the average overlap ratios of $83.6 \%$ in the whole tumor region and $82.5 \%$ in the peritumoral edema region with the manual segmentation as "ground truth".
\end{abstract}

Keywords: Automatic segmentation, brain glioma, DWI, functional MRI

\section{Introduction}

Gliomas are the most common primary tumors in the brain or spine which originate from the glial cells. Statistical results from existing studies show that gliomas account for nearly $77 \%$ of all malignant tumors in the brain, and therefore arouse great concern. The WHO has categorized gliomas into 4 grades according to pathologic evaluation of their malignancy. Due to their characteristics of infiltrative growth, different malignant grades make gliomas own a diversity of shapes and compositions in or around them. These gliomas further perform quite differently in medical imaging.

\footnotetext{
* Address for correspondence: Weibei Dou, Room 4-104, Tsinghua-Rohm Electronic Engineering Hall, Tsinghua University, Beijing 100084, China. Tel.: +86-01062781703; Fax: +86-01062770317; E-mail: douwb@tsinghua.edu.cn.
} 
For example, the low-grade astrocytoma almost appears as solid or cystic with unclear boundary to the normal tissue in conventional MRI and CT scans. But due to the rapid growth of glioblastoma multiforme, there might be some internal necrosis or bleeding accompanied by large edema surrounding the tumor.

Using a variety of medical imaging examinations [1,2] can help doctor better treat brain tumors, including preoperative localization, pathological assessment and postoperative recovery. Especially a joint analysis that combines various medical imaging modalities to represent the structural or functional characteristics of different brain tissues can provide more effective, objective and valuable diagnostic information. MRI provides a non-invasive way to characterize the microstructure and micro-dynamics of the organ or tissue in vivo. It is utilized for brain tumor diagnosis during routine examinations. In particular, 2 newcomers - DWI (Diffusion-Weighted Imaging) and fMRI (BOLD functional MRI) can provide some different potentialities than traditional T1-weighted or T2-weighted MRI. Some parameters derived from DWI, such as ADC (Apparent Diffusion Coefficient) and FA(Fractional Anisotropy) are considered to reflect not only the cytopathic effect of the glioma but also the fiber changes in its vicinity, such as displacement, destruction or infiltration. On the other hand, fMRI seems to have a better ability to reflect the dysfunction by tumor space occupying.

There have been several studies on brain tumors by MRI, some distinguish between tumors and normal tissues [3-5], some classify different tumor types [6-8] and some other grade certain tumor types $[9,10]$. In this paper, we mostly focus on detection and segmentation of gliomas in the brain. A generally recognized baseline is that the related tissues with glioma could include solid region, necrosis and peritumoral edema, which have quite different imaging representations. A variety of segmentation algorithms [11,12] has been suggested by using T1-weighted or T2-weighted MRI. Although these algorithms have the advantage of high image resolution, some arguments or problems still exist. For example, in both of the two modalities, the solid region of glioma has high similarities to cerebrospinal fluid, but significant differences with necrosis. This might lead to the failure of some algorithms based on image intensity [13] to segment the full tumor region.

In this paper, a new view of combining DWI and fMRI is attempted. A non-model segmentation scheme by multi-parameters of DWI and fMRI is proposed to distinguish the related tissues of brain glioma, including solid region, necrosis and peritumoral edema. By incorporating the knowledge of image graylevel and spatial structure, our method can achieve an automatic segmentation that does not require parameter selection and model fitting to the corresponding regions. Therefore, it has the advantages of efficiency and universality.

\section{Materials}

\subsection{Data acquisition}

The data from 10 patients diagnosed with brain glioma, provided by Beijing Tiantan Hospital, is used in this study. Their DWI and resting-state BOLD fMRI data were acquired by a Siemens 3.0T MR system with the following scanning parameters.

DWI: diffusion sensitization of $b=1000 \mathrm{~s} / \mathrm{mm}^{2}$; gradient field directions of 64 ; repetition and echo times of TR/TE $=3600 / 95 \mathrm{~ms}$; matrix size $=128 \times 128$; number of slice $=25$; image resolution $=1.80$ $\mathrm{mm} \times 1.80 \mathrm{~mm} \times 5.20 \mathrm{~mm}$.

fMRI: repetition and echo times of TR/TE $=2070 / 30 \mathrm{~ms}$; matrix size $=64 \times 64$; number of slice $=32$; image resolution $=3.75 \mathrm{~mm} \times 3.75 \mathrm{~mm} \times 5.00 \mathrm{~mm}$; time sequence length of 240 . 


\subsection{Image preprocessing}

The preprocessing steps of the raw DWI data include brain skull removing, head correction and tensor eigenvalue calculation. These were performed by the software DTI Studio (https://www.mristudio.org/wiki/installation). Next, the three related parameters [14] required were obtained as follows:

1) Apparent Diffusion Coefficient (ADC)

$$
A D C=\langle D\rangle=\frac{1}{3}\left(\lambda_{1}+\lambda_{2}+\lambda_{3}\right)
$$

2) Fractional Anisotropy (FA)

$$
\begin{aligned}
F A & =\sqrt{\frac{3}{2}} \cdot \sqrt{\frac{\left(\lambda_{1}-\langle D\rangle\right)^{2}+\left(\lambda_{2}-\langle D\rangle\right)^{2}+\left(\lambda_{3}-\langle D\rangle\right)^{2}}{\left(\lambda_{1}^{2}+\lambda_{2}^{2}+\lambda_{3}^{2}\right)}} \\
& =\sqrt{\frac{\left(\lambda_{1}-\lambda_{2}\right)^{2}+\left(\lambda_{2}-\lambda_{3}\right)^{2}+\left(\lambda_{3}-\lambda_{1}\right)^{2}}{2\left(\lambda_{1}^{2}+\lambda_{2}^{2}+\lambda_{3}^{2}\right)}}
\end{aligned}
$$

3) Trace-Weighted (TraceW)

$$
\operatorname{Tr} W=\left(S_{\lambda_{1}} S_{\lambda_{2}} S_{\lambda_{3}}\right)^{1 / 3}=S_{0} \cdot \exp (-\mathrm{b}\langle D\rangle)
$$

where $\lambda_{1}, \lambda_{2}, \lambda_{3}$ denote the eigenvalues, and $S_{0}$ denotes the original signal without diffusion sensitization.

The preprocessing steps of the fMRI data include slice timing and realigning, which were performed by the software SPM8 (http://www.fil.ion.ucl.ac.uk/spm/software/spm8). Then the time mean of fMRI sequence is used in this paper:

$$
R=\frac{1}{\mathrm{~T}} \sum_{t=1}^{\mathrm{T}} I_{\text {fMRI }}(t)
$$

where $I_{f M R I}(t)$ denotes the $t^{\text {th }}$ sample of fMRI signal, and T is the length of the total sequence.

As the X-Y space resolution of the DWI image is higher, the fMRI image is co-registered to its image space. To facilitate the following presentation, for a given voxel, $v=(x, y, z)$ is used as its coordinates in the unified space. And italic capital letters $A(v), F(v)$ and $T(v)$ denote the corresponding values of ADC, FA and TraceW, while $R(v)$ denotes the time mean of fMRI signal. All these parameters are finally normalized to the range $[0,1]$.

\section{Method}

\subsection{Overview of the proposed algorithm}

Based on the above analysis of parameter characteristics, this paper proposes a non-model automatic segmentation scheme that combines DWI and fMRI. The entire workflow of glioma tissue segmentation is illustrated in Figure 1. After the two modalities of MRI images, they are preprocessed 


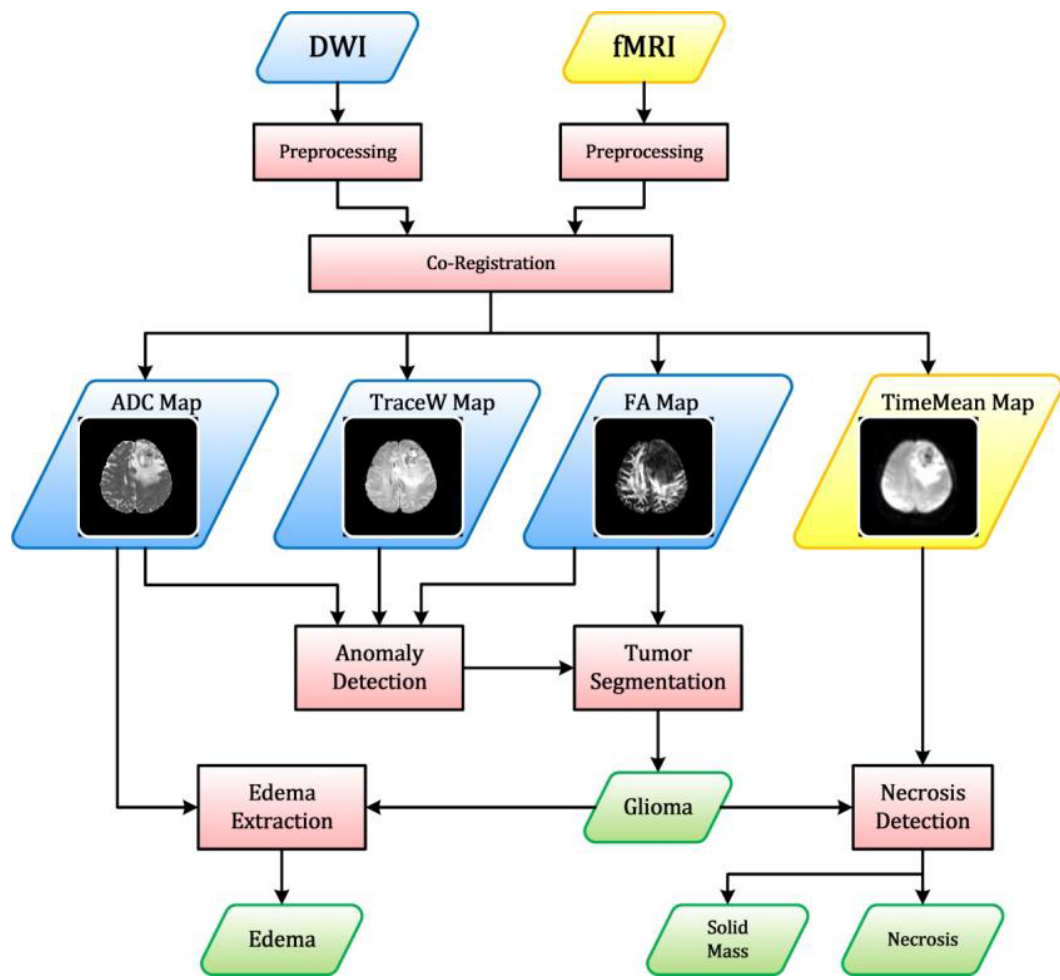

Fig. 1. Segmentation flowchart of brain glioma tissues.

singly and co-registered. The 3 DWI parameters (ADC, FA and TraceW) are calculated and utilized jointly for glioma detection and segmentation. Then, the entire tumor region is evaluated in the presence of internal necrosis by fMRI. If it is yes, then the tumor is further subdivided into necrosis region and solid region. On the other side, the topological information of the tumor region is also provided for the next peritumoral edema extraction by ADC.

\subsection{Glioma segmentation using DWI multi-parameters}

\subsubsection{Bi-normalized probability mapping}

Here a definition of bi-normalized probability mapping is given at first. Taking ADC for example, the distribution histogram of the whole brain voxels is counted:

$$
H_{A}(n)=\#\{A(v) \mid(n-1) / \mathrm{N}<A(v) \leq n / \mathrm{N}\}, n=1,2, \ldots, \mathrm{N}
$$

Then the bi-normalized probability mapping can be calculated:

$$
P_{A}(v)= \begin{cases}\frac{A(v)-a_{0}}{1-a_{0}}, & A(v) \geq a_{0} \\ \frac{A(v)-a_{0}}{a_{0}}, & A(v)<a_{0}\end{cases}
$$

where the boundary $a_{0}$ denotes the value with the highest probability distribution in $H_{A}(n)$ : 


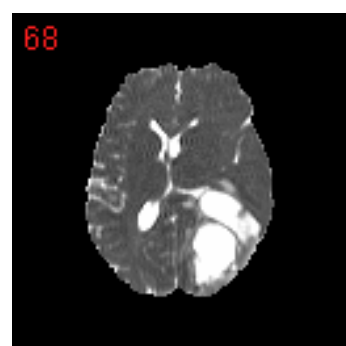

(a) ADC

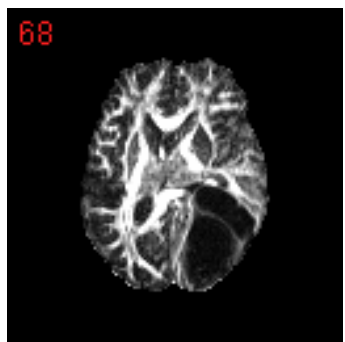

(b) FA

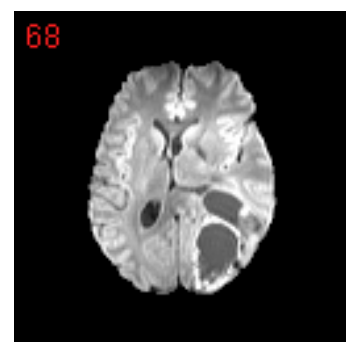

(c)TraceW

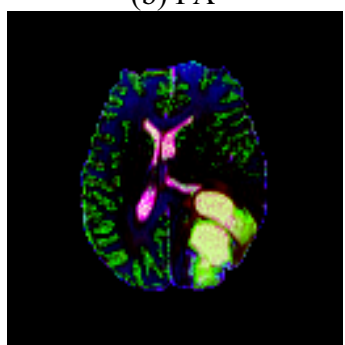

(d) Color map with positive $P_{A}(v)$, negative $P_{F}(v)$ and negative $P_{T}(v)$

Fig. 2. Color mapping of bi-normalized DWI parameters.

$$
a_{0}=\frac{1}{\mathrm{~N}} \arg \max _{1 \leq n \leq \mathrm{N}} H_{A}(n)-\frac{1}{2 \mathrm{~N}}
$$

Similarly, the probability function of FA and TraceW can be also obtained as $P_{A}(v)$ and $P_{T}(v)$.

For CSF and the solid region of glioma, their voxels mostly belong to a joint distribution range of positive $P_{A}(v)$, negative $P_{F}(v)$ and negative $P_{T}(v)$. If a RGB image is used to map their corresponding values, the mapping effect is given in Figure 2.

\subsubsection{Region growing in $F A$}

In Figure 2, it is obviously seen that the solid region of glioma is nearly yellow while CSF trends to be pink. So a rough region of the solid region is obtained:

$$
\overline{\mathrm{S}}=\left\{v \mid P_{A}(v)>0.5 \& P_{F}(v)<P_{T}(v)\right\}
$$

$\mathrm{C}_{i}(\overline{\mathrm{S}})$ is defined as the $i^{\text {th }}$ topological connected component of the voxel set $\overline{\mathrm{S}}$ sorted in descending size of each component. In this way, the largest one is selected as the seed of the next region growing: $\overline{\mathrm{G}}_{0}=\mathrm{C}_{1}(\overline{\mathrm{S}})$. Then the algorithm is described as the following steps:

Input: the FA mapping image $F(v)$; the seed voxel set $\overline{\mathrm{G}}_{0}$; the stopping threshold $\operatorname{th} 1$ Output: the voxel set of the whole glioma $\overline{\mathrm{G}}$

Steps:

1) Initialize: $\bar{G}=\bar{G}_{0}$

2) Get new neighbor voxel set of $\bar{G}$ :

$$
\overline{\mathrm{G}}_{\mathrm{N}}=\{v \mid v \notin \overline{\mathrm{G}} \text { and } \mathrm{N}(v) \cap \overline{\mathrm{G}} \neq \varnothing\}
$$

where $\mathrm{N}(v)$ denotes the neighbor set of voxel $v$. 
3) Find new voxels that meet these 2 conditions below from the set $\bar{G}_{N}$, and add them into the set $\overline{\mathrm{G}}$.

a. $F(v)<\operatorname{thr} 1$

b. $\exists \mathrm{N}(v) \in \overline{\mathrm{G}}_{\mathrm{N}}$ and $F(\mathrm{~N}(v))<\operatorname{thr} 1$

4) Repeat the above steps 2 and 3 until matching one of the stopping conditions.

a. $\# \overline{\mathrm{G}}_{\mathrm{N}}<\mathrm{N}_{0}$

b. Exceeding the maximum number of iterations

A suggested way to determine the range of $t h r 1$ is provided: $\operatorname{thr} 1 \in\left[a_{\overline{\mathrm{G}}_{0}}+1.5 \delta_{\mathrm{A}}, a_{\overline{\mathrm{G}}_{0}}+2.5 \delta_{\mathrm{A}}\right]$. Let $a_{\overline{\mathrm{G}}_{0}}$ be the value with the highest probability distribution of $A(v)$ in seed voxel set $\overline{\mathrm{G}}_{0}$, and $\delta_{\mathrm{A}}$ be the average bias of $A(v)$ lower than $a_{\overline{\mathrm{G}}_{0}}$ in $\overline{\mathrm{G}}_{0}$ :

$$
\delta_{\mathrm{A}}=\sqrt{\frac{\sum_{v \in \overline{\mathrm{G}}_{\mathrm{A}}^{\mathrm{L}}}\left[A(v)-a_{\overline{\mathrm{G}}_{0}}\right]^{2}}{\#\left\{\overline{\mathrm{G}}_{\mathrm{A}}^{\mathrm{L}}\right\}}}, \quad \overline{\mathrm{G}}_{\mathrm{A}}^{\mathrm{L}}=\left\{v \in \overline{\mathrm{G}}_{0} \mid A(v) \leq a_{\overline{\mathrm{G}}_{0}}\right\}
$$

\subsection{Necrosis detection}

When the glioma deteriorates to a certain extent, some cells appear to stop metabolism and lose function, which is called as necrosis. This is an important indicator of glioma malignancy and is reflected as dark area in the fMRI signal. Therefore, the algorithm of necrosis detection is:

Input: the ADC probability mapping $P_{A}(v)$; the glioma voxel set $\overline{\mathrm{G}}$; the threshold $t h r 2$ and thr3

Output: the voxel set of the necrosis $\bar{N}$

\section{Steps:}

1) Find the candidate voxel set of necrosis:

$$
\overline{\mathrm{N}}_{0}=\left\{v \mid v \in \overline{\mathrm{G}} \text { and } P_{A}(v)<t h r 2\right\}
$$

2) Initialize the necrosis set as null: $\overline{\mathrm{N}}=\varnothing$

3) While connected component exists:

If $\exists v \in \mathrm{C}_{i}\left(\overline{\mathrm{N}}_{0}\right)$ satisfies $R(v)<t h r 3$, then

$i=i+1$;

$$
\overline{\mathrm{N}}=\overline{\mathrm{N}} \cup \mathrm{C}_{i}\left(\overline{\mathrm{N}}_{0}\right)
$$

The range of $t h r 2$ can be obtained similar to thr $1: t h r 2 \in\left[p_{\overline{\mathrm{G}}_{0}}-2.5 \delta_{\mathrm{P}_{\mathrm{A}}}, p_{\overline{\mathrm{G}}_{0}}-1.5 \delta_{\mathrm{P}_{\mathrm{A}}}\right]$. Let $p_{\overline{\mathrm{G}}_{0}}$ be the value with the highest probability distribution of $P_{A}(v)$ in seed voxel set $\overline{\mathrm{G}}_{0}$, and $\delta_{\mathrm{P}_{\mathrm{A}}}$ be the average bias of $P_{A}(v)$ higher than $p_{\overline{\mathrm{G}}_{0}}$ in $\overline{\mathrm{G}}_{0}$. And the threshold $t h r 3$ is an empirical value using the maximum of the average $R(v)$ in necrosis of 12 patients, which is 0.32 in our experiment. After this, the rest of $\overline{\mathrm{G}}$ is considered as the solid region:

$$
\overline{\mathrm{S}}=\overline{\mathrm{G}}-\overline{\mathrm{N}}=\left\{v \in \overline{\mathrm{G}} \mid A(v)>a_{0}\right\}
$$

\subsection{Peritumoral edema extraction}


Another related diseased tissue that always occurs around the tumor is edema. Previous studies have shown that the ADC value in edema is between WM and CSF with significant differences. By utilizing the features in topological structure and value information, the edema region can be identified as:

$$
\overline{\mathrm{E}}=\mathrm{C}_{1}(\{v \mid \operatorname{thr} 4<A(v)<\operatorname{thr} 5\} \cup \overline{\mathrm{G}})-\overline{\mathrm{G}}
$$

Tests performed in all the patients revealed that the fixed values for $t h r 4$ and $t h r 5$ could make better segmentations than some adaptive values. Their referential ranges are also provided here: $t h r 4 \in$ $[0.25,0.30]$, thr $5 \in[0.45,0.65]$

\section{Results and discussion}

\subsection{Experimental results}

We use the proposed method to segment the corresponding diseased regions in 10 patients with gliomas. In some cases that have small tumor region, the thresholds based on histogram may result in failures of the segmentation. So the following group of values is provided as default that has been checked to be feasible in all patients:

$$
\text { thr } 1=0.09, \text { thr } 2=0.45, \operatorname{thr} 3=0.32, \text { thr } 4=0.28, \operatorname{thr} 5=0.49
$$

The proposed method is compared with other methods, such as an automatic method using fuzzy model [13], and two semi-automatic methods using active contour model [15] and GICOV-DP snake

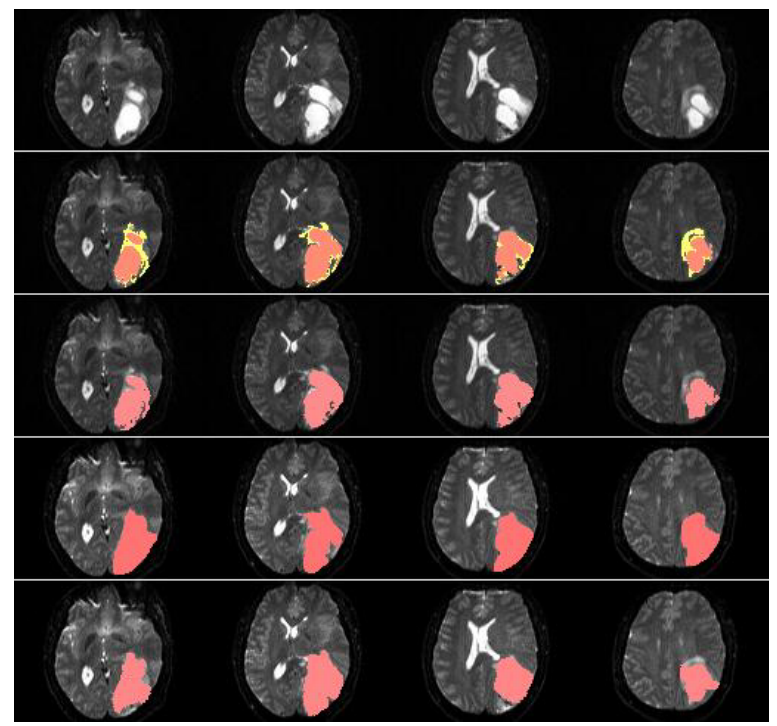

(a) Sub.1 with no necrosis in glioma and slightly edema

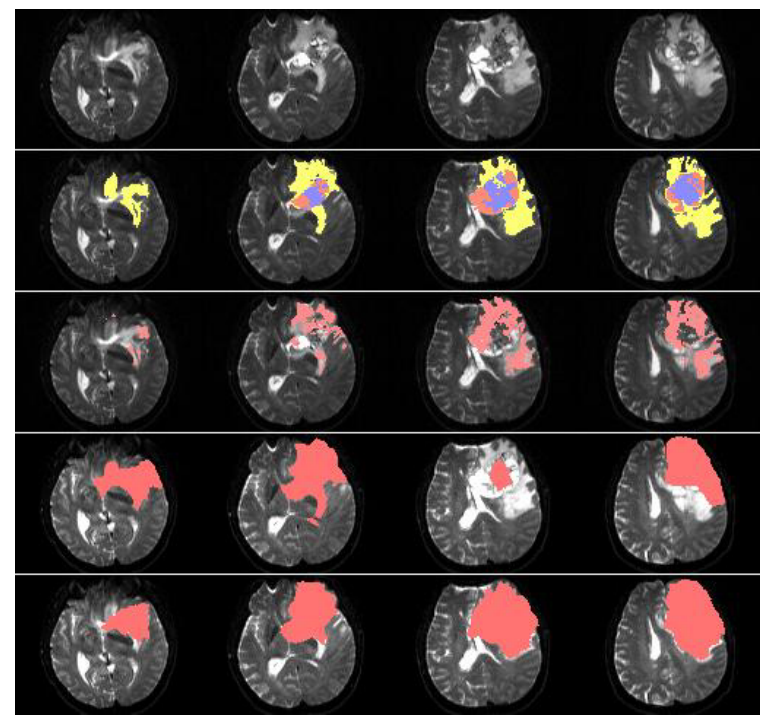

(b) Sub.2 with necrosis in glioma and massive edema

Fig. 3. Segmentation results of the proposed method and other comparative methods. Marking colors: red - solid region; blue - necrosis region; yellow - peritumoral edema. Rows: 1 - DWI b0 image; 2 - segmentation of the proposed method; 3 segmentation of the method [13]; 4 - segmentation of the method [15]; 5 - segmentation of the method [16]. 
[16]. The segmentation results of 2 patients are shown in Figure 3. It is seen that the 2 reference methods would only handle the case in which the glioma only has the solid mass, but fail if there is some necrosis. Besides, they could not reach a subdivision of the extracted glioma region. On the other side, our method can solve the problems and achieve more accurate results.

For a more comprehensive evaluation, we make a comparison between the proposed segmentation and the manual segmentation using the following criteria:

1) Ratio of Overlap ( $\left.\mathrm{R}_{\mathrm{OLP}}\right)[3]$ :

$$
\mathrm{R}_{\mathrm{OLP}}=\frac{\#\{\mathrm{~S} \cap \mathrm{R}\}}{\#\{\mathrm{~S} \cup \mathrm{R}\}}
$$

where the set $\mathrm{S}$ denotes the voxel set of one segmented region using our proposed method, and the set $\mathrm{R}$ denotes the one using manual segmentation.

2) Hausdorff Distance $\left(D_{H}\right)[3]$ :

$$
\mathrm{D}_{\mathrm{H}}=\max \left\{\sup _{u \in \mathrm{S}} \inf _{v \in \mathrm{R}} \mathrm{d}(u, v), \sup _{v \in \mathrm{S}} \inf _{u \in \mathrm{R}} \mathrm{d}(u, v)\right\}
$$

where sup and inf represent the supremum and the infimum, $\mathrm{d}(u, v)$ denotes the Euclidean distance between voxel $u$ and $v$.

3) Root Mean Squared Error (RMSE)[15]:

$$
\operatorname{RMSE}=\sqrt{\frac{\sum_{u \in \mathrm{S}_{\mathrm{c}}}\left[i n f_{\left.v \in \mathrm{R}_{\mathrm{c}} \mathrm{d}(u, v)\right]^{2}}\right.}{\#\left\{\mathrm{~S}_{\mathrm{c}}\right\}}}
$$

where $S_{c}$ and $R_{c}$ are the contours of $S$ and $R$.

Two regions of the whole tumor and the peritumoral edema are selected for the quantitative evaluation in 6 patients, and the results are listed in Table 1. Both $D_{\mathrm{H}}$ and RMSE are in voxel units. An image result of patient Sub.3 is given in Figure 4.

\subsection{Discussion}

Major MRI modalities in the current literature for brain tumor segmentation are focused on traditional T1-weighted or T2-weighted MR images. However, a common problem associated with these studies is the failure or insufficiency to fully segment the related regions of the tumor. In this

Table 1

Comparison results with the manual segmentation

\begin{tabular}{l|lll|lll}
\hline Subject & \multicolumn{3}{|l|}{ Whole Tumor Region } & & \multicolumn{3}{c}{ Peritumoral Edema Region } \\
No & $\mathbf{R}_{\text {OLP }}$ & $\mathbf{D}_{\mathbf{H}}$ & $\mathbf{R M S E}$ & $\mathbf{R}_{\text {OLP }}$ & $\mathbf{D}_{\mathbf{H}}$ & RMSE \\
\hline Sub.1 & 0.834 & 6.32 & 1.48 & 0.816 & 6.08 & 1.43 \\
Sub.2 & 0.951 & 1.41 & 0.25 & 0.690 & 8.54 & 1.62 \\
Sub.3 & 0.950 & 4.12 & 0.81 & 0.889 & 15.52 & 2.51 \\
Sub.4 & 0.850 & 6.32 & 1.06 & 0.886 & 6.00 & 1.14 \\
Sub.5 & 0.838 & 4.00 & 1.83 & 0.828 & 13.45 & 2.17 \\
Sub.6 & 0.595 & 17.42 & 5.92 & 0.840 & 9.22 & 3.23 \\
Average & $\mathbf{0 . 8 3 6}$ & $\mathbf{6 . 6 0}$ & $\mathbf{1 . 8 9}$ & $\mathbf{0 . 8 2 5}$ & $\mathbf{9 . 8 0}$ & $\mathbf{2 . 0 2}$ \\
\hline
\end{tabular}




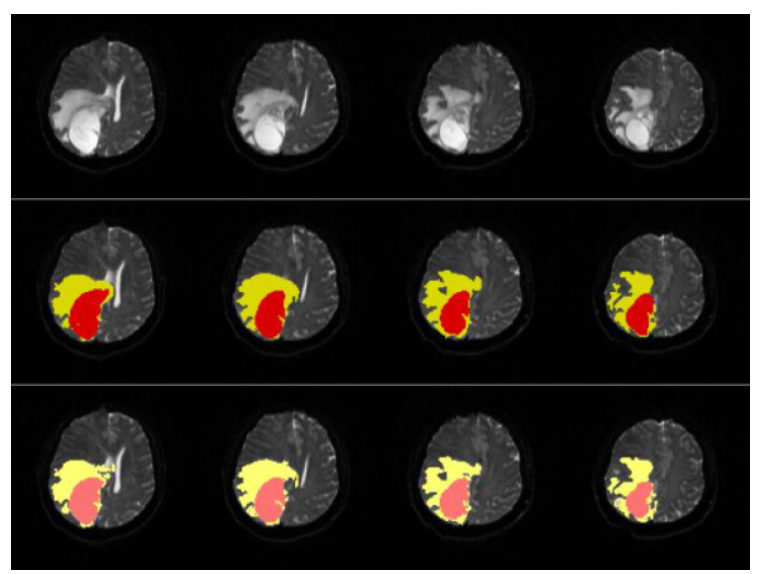

Fig. 4. Comparison result of Sub.3 with the manual segmentation. Marking colors: red - solid region; yellow - peritumoral edema. Rows: 1 - DWI b0 image; 2 - the manual segmentation; 3 - the proposed method.

paper we utilized different MRI modalities to compensate for the above-mentioned weakness. The results in Figure 3 demonstrated two advantages of our work. First, in the cases without necrosis (see Sub.1), our method can efficiently differentiate between the tumor cells and the edema. This feature is unavailable in the other comparative methods. Second, when there is necrosis in the tumor (see Sub.2), our method utilize the consistent low FA values in both tumor solid and necrosis, to obtain the complete area. Moreover, combining these results with fMRI we can pinpoint the dysfunctional part in glioma, which is not available in structural MR images.

However, there are some limitations in the proposed method. The present image resolutions of DWI and fMRI can't reach the T1-weighted or T2-weighted MRI and some details are missing. Another problem is that our method may fail when the size of gliomas too small to be distinct from noises. The robustness of the threshold selection in some steps also needs to be verified by testing additional samples. The purpose for studying glioma segmentation in the perspective of DWI and fMRI is not to replace the routine protocols, but to provide a supplement to improve the diagnosis of glioma. Our work is also easy to extend with other imaging modalities.

\section{Conclusion}

This paper describes morphological analysis of tumors from 2 MRI modalities of DWI and fMRI, and proposes a non-model automatic segmentation scheme of the pathological regions with brain glioma. Our approach utilizes preexisting knowledge of imaging intensity and combines it with topological structure in the brain tissues. A promising advantage of our work is to provide an effective and adequate subdivision of the related pathological regions with glioma, which could compensate for the vulnerability of the traditional T1-weighted or T2-weighted MRI to some extent. By testing real data of patients with glioma and using the manual segmentation as "ground truth", the average overlap ratios of the proposed approach have reached $83.6 \%$ in the whole tumor region and $82.5 \%$ in the peritumoral edema region. The average Hausdorff distances are 6.60 voxels for the whole tumor region and 9.80 voxels for the peritumoral edema region. In addition, it would be considered as a helpful and necessary procedure for other further researches, such as grading of gliomas, and analyzing changes of peritumoral fibers or influences on brain functional activities. 


\section{Acknowledgment}

We thank Beijing Tiantan Hospital, Capital Medical University for providing the clinical data, for guiding this study and for suggestions in manual segmentation as "ground truth". This work was supported by the projects of NSFC-61171002, NSFC-60372023 and Tsinghua University Initiative Scientific Research Program 20131089382.

\section{References}

[1] A. Mert, B. Kiesel, A. Wohrer, M.M. Moreno, G. Minchev, J. Furtner, E. Knosp, S. Wolfsberger and G. Widhalm, Introduction of a standardized multimodality image protocol for navigation-guided surgery of suspected low-grade gliomas, Neurosurgical Focus 38 (2015), E4.

[2] G. Zoccatelli, F. Alessandrini, A. Beltramello and A. Talacchi, Advanced magnetic resonance imaging techniques in brain tumours surgical planning, Journal of Biomedical Science and Engineering 6 (2013), 403-417.

[3] D. Cobzas, N. Birkbeck, M. Schmidt, M. Jagersand and A. Murtha, 3D variational brain tumor segmentation using a high dimensional feature set, IEEE International Conference on Computer Vision, Rio de Janeiro, Brazil, 2007, pp. 1-8.

[4] T.L. Jones, T.J. Byrnes, G. Yang, F.A. Howe, B.A. Bell and T.R. Barrick, Brain tumor classification using the diffusion tensor image segmentation (D-SEG) technique, Neuro-Oncology 7 (2015), 466-476.

[5] C. Vijayakumar, G. Damayanti, R. Pant and C.M. Sreedhar, Segmentation and grading of brain tumors on apparent diffusion coefficient images using self-organizing maps, Computerized Medical Imaging and Graphics 31 (2007), 473-484.

[6] S. Lu, D. Ahn, G. Johnson and S. Cha, Peritumoral diffusion tensor imaging of high-grade gliomas and metastatic brain tumors, American Journal of Neuroradiology 24 (2003), 937-941.

[7] S. Wang, S. Kim, S. Chawla, R.L. Wolf, W. Zhang, D.M. O'Rourke, K.D. Judy, E.R. Melhem and H. Poptani, Differentiation between glioblastomas and solitary brain metastases using diffusion tensor imaging, Neuroimage 44 (2009), 653-660.

[8] R. Jiang, F. Du, C. He, M. Gu, Z. Ke and J. Li, The value of diffusion tensor imaging in differentiating high-grade gliomas from brain metastases: a systematic review and meta-analysis, PLoS One 9 (2014), e112550.

[9] T. Inoue, K. Ogasawara, T. Beppu, A. Ogawa and H. Kabasawa, Diffusion tensor imaging for preoperative evaluation of tumor grade in gliomas, Clinical Neurology and Neurosurgery 107 (2005), 174-180.

[10] H.Y. Lee, D.G. Na, I.C. Song, D.H. Lee, H.S. Seo, J. Kim and K.H. Chang, Diffusion-tensor imaging for glioma grading at 3-T magnetic resonance imaging: analysis of fractional anisotropy and mean diffusivity, Journal of Computer Assisted Tomography 32 (2008), 298-303.

[11] S. Bauer, R. Wiest, L.P. Nolte and M. Reyes, A survey of MRI-based medical image analysis for brain tumor studies, Physics in Medicine Biology 58 (2013), 97-129.

[12] N. Gordillo, E. Montseny and P. Sobrevilla, State of the art survey on MRI brain tumor segmentation, Magnetic Resonance Imaging 31 (2013), 1426-1438.

[13] W. Dou, S. Ruan, Y. Chen, D. Bloyet and J.M. Constans, A framework of fuzzy information fusion for the segmentation of brain tumor tissues on MR images, Image and Vision Computing 25 (2007), 164-171.

[14] D.L. Bihan, J.F. Mangin, C. Poupon, C.A. Clark, S. Pappata, N. Molko and H. Chabriat, Diffusion tensor imaging: Concepts and applications, Journal of Magnetic Resonance Imaging 13 (2001), 534-546.

[15]L. Wang, L. He, A. Mishra and C. Li, Active contours driven by local Gaussian distribution fitting energy, Signal Processing 89 (2009), 2435-2447.

[16] N. Ray, S.T. Acton and H. Zhang, Seeing through clutter: Snake computation with dynamic programming for particle segmentation, Proceeding of International Conference on Pattern Recognition, Tsukuba, Japan, 2012, pp. 801-804. 